\title{
Independent sets, codes and their properties
}

\author{
Meixia TANG ${ }^{1, \mathrm{a}}$, Yu LIU ${ }^{2, \mathrm{~b}}$ \\ ${ }^{1}$ School of Information Engineering, Nanning College for Vocational \\ Technology, Nanning, Guangxi \\ ${ }^{2}$ Modern Education Technology Center, Nanning College for Vocational \\ Technology,Nanning,Guangxi \\ asmiletmx@126.com, ${ }^{b}$ ly102091002@163.com
}

\begin{abstract}
.
The concept that strict binary relation on free monoids is introduced, some characterizations for strict binary relations is given, the ordering properties of the set of all strict binary relations as well as some subsets of it are exhibited. Moreover, it is proved that the independent languages of co-compatible quasi-strict relations are codes.
\end{abstract}

Keywords: code; independent set; quasi-strict binary relation; co-compatible binary relation

\section{Introduction}

This paper will introduce the concept of quasi-strict relations, some order relations and codes, and discuss the relationship between independent language of quasi-strict relations and code. At last prove a nonempty co-compatible quasi-strict relation independent sets are code.

\section{Basic notions and notation}

Let $X$ be an alphabet and let $X^{*}$ be the free monoid generated by $X$. Any element of $X^{*}$ is called a word over $X$ and any subset $A$ of $X^{*}$ is called a language over $X$. Let $X^{+}=X^{*}-\{1\}$, where 1 is the empty word. We let $\lg (w)$ denote the length of the word $w$. For any $A, B \subseteq X^{*}$, 
let $A B=\{a b \mid a \in A, b \in B\}$. A non-empty language $A \subseteq X^{+}$is called a code if $a_{1} a_{2} \cdots a_{n}=b_{1} b_{2} \cdots b_{m}, a_{i}, b_{j} \in A, i=1,2, \cdots, n, j=1,2, \cdots, m$, implies $n=m_{\text {and }} a_{i}=b_{i}$ for $i=1,2, \cdots, n$. A code $A$ is said to be a prefix (suffix) code if $A \cap A X^{+}=\phi\left(A \cap X^{+} A=\phi\right)$.

A binary relation $\rho$ on $X^{*}$ is a subset of $X^{*} \times X^{*}$.

A binary relation $\rho$ defined on a set $A_{\text {is called a partial order relation if for }}$ all a, b, and c in $A$, we have that:

(i) a $\rho$ a (reflexivity);

(ii) if a $\rho_{\mathrm{b} \text { and } \mathrm{b}} \rho_{\mathrm{a}}$ then $\mathrm{a}=\mathrm{b}$ (antisymmetry);

(iii) if a $\rho_{\mathrm{b} \text { and b }} \rho_{\mathrm{c}}$ then a $\rho_{\mathrm{c}}$ (transitivity).

The set $A_{\text {is called a partially ordered set, poset for short, denoted }}$ by $(A, \rho)$.

We call $\rho$ a quasi-strict binary relation on $X^{*}$ if for all $a, b \in X^{*}$,

(i) $(a, a) \in \rho$ and $(1, a) \in \rho$

(ii) $(a, b) \in \rho$ and $\lg (a)=\lg (b)_{\text {implies }} a=b$

It is clear that $\rho_{\text {is reflexive. }}$

We call $\rho$ a strict binary relation on $X^{*}$ if for all $a, b \in X^{*}$,

(i) $(a, a) \in \rho$ and $(1, a) \in \rho$

(ii) $(a, b) \in \rho$ implies $\lg (b) \geq \lg (a)$

(iii) $(a, b) \in \rho$ and $\lg (a)=\lg (b)_{\text {implies }} a=b$

It is clear that $\rho$ is reflexive, antisymmetric and transitive.

We let $\omega_{\rho}$ denote the relation $\rho \cup \rho^{-1}\left(\rho^{-1}\right.$ is the Inverse relation of $\rho$ ), obviously it is reflexive and symmetric. 
Let $A_{1}$ be any subset of a set $A_{2}$ with a partial ordering $\leq$ on $A_{2}$. An element $m$ is said to be a maximal element in $A_{1}$ if for every $a \in A_{1}$, $m \leq a_{\text {implies }} a \leq m$. The minimal elements of $A_{1}$ is defined correspondingly.

A non-empty subset $D_{\text {of }} X^{*}$ is said to be dependent with respect to a binary relation $\rho$ defined on $X^{*}$ or simply $\rho_{\text {-dependent if there exist two }}$ distinct words $u$ and $v$ in $D$ such that $u \rho v$. As an exception, let $\{1\}$ be $\rho$-dependent for every $\rho$. A set $H \subseteq X^{*}$ is said to be $\rho$-independent whenever $H$ is not $\rho$-dependent. The family of all $\rho$-dependent ( $\rho$-independent) subsets of $X^{*}$ is called a $\rho$-dependence ( $\rho$-independence) in $X^{*}$ and denoted by $D_{\rho}\left(H_{\rho}\right.$, respectively). Every word $\neq 1$ is in $H_{\rho}$ for every $\rho$.

A $\rho$-dependence relation denoted by $\stackrel{\rho}{\propto}$ is a binary relation defined on $X^{*}$ such that $\stackrel{\rho}{\propto} v$ if and only if $u \rho v$ or $v \rho u$. The symbol $u \stackrel{\rho}{\propto} v$ means $u \tilde{\rho} v_{\text {and }} v \tilde{\rho} u$.

Let $M$ be a $\rho$-independent subset of $S$. Then $M$ is called a maximal $\rho$-independent subset of $S$ if $M \bigcup\{x\}$ is $\rho$-dependent for all $X \in S \backslash M$.

\section{Some order relations and codes}

We define now the following strict binary relations on $X^{*}$ :

(i) $\rho_{P}=\left\{(u, u x) \mid u \in X^{*}, x \in X^{*}\right\}$ 


$$
\begin{aligned}
& \text { (ii) } \rho_{s}=\left\{(u, x u) \mid u \in X^{*}, x \in X^{*}\right\} . \\
& \text { (iii) } \rho_{d}=\left\{(u, y) \mid y=u x \text { and } y=w u_{\text {for some }} x, w \in X^{*}\right\} . \\
& \text { (iv) } \rho_{c}=\left\{(u, y) \mid y=u x=x u \text { for some } x \in X^{*}\right\} . \\
& \text { (v) } \rho_{e=}\left\{(u, y) \mid u=u_{1} u_{2} \cdots u_{n}, y=y_{1} u_{1} y_{2} u_{2} \cdots y_{n} u_{n} y_{n+1}\right. \text {, for some } \\
& \left.n \geq 0 \text {,and } u_{i}, y_{j} \in X^{*}\right\} . \\
& \text { (vi) } \rho_{u}=\{(u, y) \mid u=y \text { or } \lg (u)<\lg (y)\} . \\
& \text { (vii) } \rho_{b}=\left\{(u, y) \mid y=u x \text { or } y=w u \text { for some } x, w \in X^{*}\right\} . \\
& \text { (viii) } \rho_{i}=\left\{(u, y) \mid y \in X^{*} u X^{*}\right\} . \\
& \text { (ix) } \rho_{o}=\left\{(u, y) \mid u=u_{1} u_{2} \text { and } y \in u_{1} X^{*} u_{2}\right\} . \\
& \text { In general } \rho_{c} \subset \rho_{d} \subset \rho_{p} \subset \rho_{i} \subset \rho_{e} \subset \rho_{u} \text { and } \rho_{d} \subset \rho_{s} \subset \rho_{i} \text {.It is }
\end{aligned}
$$
easy to see that the class of all prefix codes, all hypercodes, all infix codes, all bifix codes, all outfix codes and the class of all suffix codes over $X$ are exactly the class of all independent sets of $\rho_{p}, \rho_{e}, \rho_{i}, \rho_{b}, \rho_{o}$ and $\rho_{s}$ respectively. Obviously, the strict binary relations $\rho_{p}, \rho_{s}, \rho_{e}, \rho_{d}, \rho_{u}, \rho_{b}, \rho_{i}, \rho_{o}$ and $\rho_{c}$ are partial orders on $X^{*}$.

PROPOSITION1. Let $X$ be an alphabet and let $x, y \in X^{+}$, $\lg (y) \geq \lg (x)$.Then the following are equivalent:

$$
\begin{aligned}
& \text { (i) } y=u x=x u \text { for some } u \in X^{*} \text {. } \\
& \text { (ii) } x y=y x \text {. } \\
& \text { (iii) } x=w^{n}, y=w^{n+r} \text {, where } n \geq 1, r \geq 0 \text { and } w \text { is a primitive }
\end{aligned}
$$
word over $X$. 
(iv) $\{x, y\}$ is not a code.

PROPOSITION2. If $X$ contains more than one element, then there is no strict binary relation $\rho$ defined on $X^{*}$ such that the class of all independent sets is exactly the class of all codes over $X$.

PROOF: Let $X=\{a, b, \cdots\}$ where $a \neq b$. Suppose $\rho$ is a strict binary relation such that the class of all independent sets is exactly the class of all codes. Since every prefix code and every suffix code is a code, we can conclude that $\rho \subseteq \rho_{p}$ on $X^{+}$and $\rho \subseteq \rho_{s}$ on $X^{+}$. It follows that for all $u, v \in X^{+}, \quad(u, v) \in \rho$ implies that $v=u x$ and $v=y u$ for some $x, y \in X^{*}$. The set $A=\left\{a b^{2}, b a, a b, b^{2} a\right\}$ is not a code, because $a b^{2} \cdot b a=a b \cdot b^{2} a$. However $A$ is an independent set with respect to $\rho$, a contradiction!

Proposition3. If $A$ is a code over $X$, then $A$ is an independent set with respect to $\rho_{c}$.

PROOF: Let $A$ be a code. The case when $A$ contains only one word is trivial. Now let $u, v \in A_{\text {such that }}(u, v) \in \rho_{c}, u \neq v$.Then by definition $v=u x=x u$ for some $x \in X^{+}$.We have $u v=u(x u)=(u x) u=v u$.This contradicts the fact that $A$ is a code. Hence $A$ is an independent set with respect ${ }_{\text {to }} \rho_{c}$.

An independent set with respect to $\rho_{d}$ may not be a code. For example let $\mathrm{X}=\{\mathrm{a}, \mathrm{b}\}$. Then $A=\left\{a b^{2}, b a, a b, b^{2} a\right\}$ is an independent set with respect to $\rho_{d}$ but $A$ is not a code. 
Remark that any code is $\rho_{c}$-independent . If every $\rho$-independent ${ }_{\text {set }}$ is a code, then $\rho_{c} \subseteq \rho$.

\section{Co-compatible binary relations on $X^{*}$}

Let $\rho$ be a binary relation on $X^{*}$ and let $[\rho]=\overline{\omega_{\rho}}$, i.e. $[\rho]$ is the

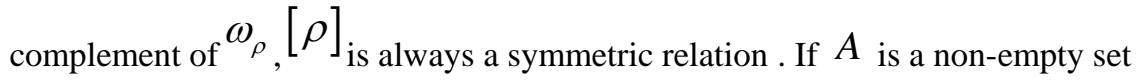
of $X^{+}$, then $A$ is $\rho$-independent if and only if $x[\rho] y$ for every $x, y \in A, x \neq y$.

A binary relation $\rho$ is said to be compatible if

(i) $(x, y) \in \rho$ and $z \in X^{*}$ imply $(x z, y z)$ and $(z x, z y) \in \rho$.

(ii) $\left(x_{1}, x_{2}\right)$ and $\left(y_{1}, y_{2}\right) \in \rho$ imply $\left(x_{1} y_{1}, x_{2} y_{2}\right) \in \rho$.

It is well known that if $\rho$ is a reflexive and transitive binary relation, then (i) is equivalent to (ii).

A binary relation $\rho$ is said to be co-compatible if and only if $[\rho]_{\text {is }}$ compatible. The strict binary relations $\rho_{p}, \rho_{s}$ and $\rho_{e}$ are co-compatible while $\rho_{d}$ is not.

PROPOSITION4. Let $\rho$ be a reflexive relation that is co-compatible. Then every $\rho$-independent set is a code.

PROOF: Since $\rho$ is a reflexive binary relation by assumption, we have $x[\not] x$ for all $x \in X^{*}$. Now let $A \subseteq X^{+}$be a $\rho$-independent set. Then $A, A^{2}, \cdots, A^{i}, \cdots$ are $\rho$-independent sets, since $\rho$ is co-compatible. 
Suppose $A$ is not a code. Then there exist $x_{i}, y_{j} \in A$ such that $x_{1} x_{2} \cdots x_{m}=y_{1} y_{2} \cdots y_{n}$ for some $m, n \geq 1$ and $x_{1} \neq y_{1}$. We have then $x_{1} x_{2} \cdots x_{m} y_{1} y_{2} \cdots y_{n}=y_{1} y_{2} \cdots y_{n} x_{1} x_{2} \cdots x_{m}$. And $z_{1}=x_{2} \cdots x_{m} y_{1} y_{2} \cdots y_{n} \neq y_{2} \cdots y_{n} x_{1} x_{2} \cdots x_{m}=z_{2} \quad$ where $z_{1}, z_{2} \in A^{m+n-1} \quad$. Hence $\quad x_{1}[\rho] y_{1} \quad$ and $\quad z_{1}[\rho] z_{2}$ and therefore $x_{1} z_{1}[\rho] y_{1} z_{2}$ holds. This is a contradiction, since $x[\not \supset] x$ for all $x \in X^{*}$.

\section{Summary}

Code is the most basic tool of information processing,coding theory is the core of formal linguistics, and is often regarded as a separate branch of theoretical computer science and combinatorial mathematics. Prefix code(especially Hoffman code and ASCII code) is the most widely used code.The judge and generation of code is the key problem of coding theory. Notice that some code (such as the prefix, suffix code, super code and infix code etc.)can be defined as a independent language of some relation on free monoids. Introduce a new question: what relation on free monoids that the independent language of it is a code? This paper discusses some properties of independent set and the code, and this problem is solved at last .

\section{References}

[1] H.J.Shyr, "Free Monoids and Languages”, Lecture Notes, Hon Min Book Company, Taichung, 2001.

[2] S.Ginsburg, "Mathematical Theory of Context-Free Languages", McGraw-Hill, New-York, 1966. 
[3] L.H.Haines, on free monoid partially ordered by embedding, J. Combina-torial Theory 6, 94-98, 1969.

[4] H.J.Shyr, and G.Thierren, Hypercodes, Information and Control, Vol. 24, N0.1, 45-54, 1974.

[5] H.K.Hsiao,Y.T.Yeh,S.S.Yu, Dependences related to strict binary relations, Theoretical Computer Science 347, 306-324, 2005.

[6] H.Jurgensen, S.S.Yu, Relations on free monoids, their independent sets, and codes, Internat. J. Comput.Math. 40, 17-46,1991.

[7] J.Berstel and D.Perrin, “Theory of Codes”,Academic Press,Version $8,2002$.

[8] S.S.Yu, "Languages and Codes”, Lecture Notes, Tsang Hai Book Publishing Co., Taichung, 2005.

[9] H.J.Shyr and G.Thierrin, Codes and Binary Relations, Lecture Notes 586,180-188, Springer-Verlag, 1976.

[10] H.Jurgensen,S.Konstantinidis, the hierarchy of codes, lecture notes in computer science; Vol.710, 50-68, 1993.

[11] H.Jurgensen, Syntactic Monoids of Codes, report327, Dep. Comp. Sci., The University of Western Ontario, 1992.

[12] H.J.Shyr, S.S.Yu, Intercodes and Some Related Properties, Soochow J. of Math., Volume 16, No.1, 95-107, 1990.

[13] John M.Howie, Automata and Languages, Clarendon Press, 1991.

[14] F. Blanchet-Sadri, Codes, orderings, and partial words, Theoretical Computer Science, Volume 329, Issue 1-3, 177-202, 2004. 